\title{
Structural Rationalization for the Nonmutagenic and Mutagenic Bypass of the Tobacco-Derived 04-4-(3-pyridyl)-4-oxobut-1-yl-thymine Lesion by Human Polymerase १: A Multiscale Computational Study
}

Priya Bhutani, ${ }^{\S}$ Makay T. Murray, ${ }^{\S}$ Craig W. Sommer, Katie A. Wilson and Stacey D. Wetmore*

Department of Chemistry and Biochemistry, University of Lethbridge, 4401 University Drive West, Lethbridge, Alberta Canada T1K 3M4

\section{Supporting Information}

Table of Contents

(11 Pages)

Figure S1: Percentage of extended, stacked and T-shaped O4-POB-T conformations identified from the

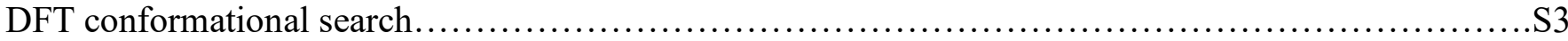

Figure S2: MD representative structure of the pol $\eta$ pre-insertion complex with a) O4-POB-T extended into the open pocket or b) stacked with the 3'-base pair.

Figure S3: Hydrogen-bonding occupancies between O4-POB-T and Q38 in the pol $\eta$ a) pre-insertion complex, and ternary complex corresponding to b) dATP or c) dGTP insertion..... S4

Figure S4: Key DFT structural parameters for the canonical T:A base pair and the associated binding

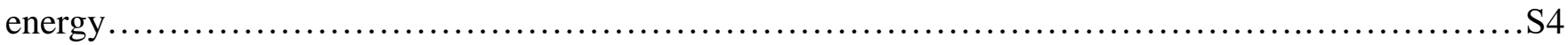

Figure S5: Overlay of X-ray crystal structure and MD representative structure for pol $\eta$ insertion of a) dATP and b) dGTP opposite O4-POB-T. S5

Figure S6: a) Definition of reaction parameters with respect to the incoming dNTP and the corresponding 5 ' base and b) $\mathrm{Mg}^{2+}$ coordination required to promote catalytic activity of pol $\eta$. .S5

Figure S7: X-ray crystal structure corresponding to pol $\eta$ insertion of dATP opposite an abasic site.....S5 Figure S8: MD representative structure of DNA duplexes containing the a) O4-POB-T:A pair or b) O4POB-T:G mispair. ..S6

Figure S9: MD representative structure corresponding to pol $\eta$ insertion of dGTP partially stabilized by a water chain when $\mathrm{O} 4-\mathrm{POB}-\mathrm{T}$ is located in the open pocket. .S6

Figure S10: MD representative structure, including hydrogen-bond occupancies, of the post-extension DNA duplex corresponding to dATP or dGTP insertion opposite a) O4-POB-T or b) O4-Me-T. S7

Figure S11: Rotation about the glycosidic bond in the canonical T nucleoside between the anti and syn conformations, as well as identification of the Watson-Crick and Hoogsteen hydrogen-bonding face....S7

Table S1: Atom types, atom names and partial charges for the O4-POB-T nucleobase used in the Hyperchem conformational search. 
Table S2: Atom names and types, as well as associated partial charges for the O4-POB-T nucleotide used

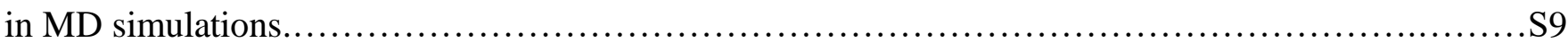

Table S3: Average backbone RMSD and associated standard deviation over MD simulations on pol $\eta$ ternary complexes and post-extension duplexes corresponding to dNTP insertion opposite O4-POB-

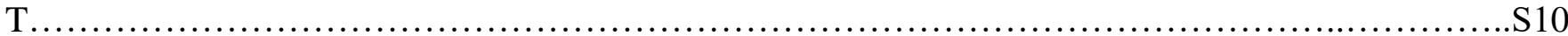

Table S4: Average distance and occupancy of $\mathrm{Mg}^{2+}$ coordinating residues throughout MD simulations on the pol $\eta$ ternary complex corresponding to dATP insertion opposite O4-POB-T....................S10

Table S5: Average base-pair hydrogen-bonding distances and angles between O4-POB-T and an opposing A or $\mathrm{G}$ over MD simulations on ternary complexes and post-extension duplexes...................S11

Table S6: Average distance and occupancy of $\mathrm{Mg}^{2+}$ coordinating residues throughout MD simulations on the pol $\eta$ ternary complex corresponding to dGTP insertion opposite O4-POB-T... 


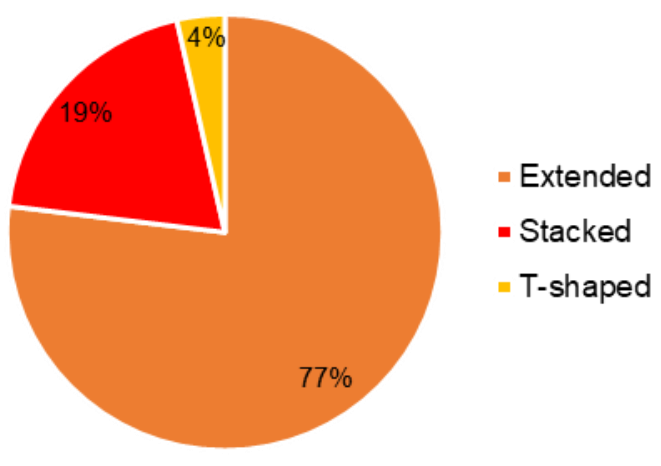

Figure S1: Percentage of extended (orange), stacked (red) and T-shaped (yellow) O4-POB-T conformations identified from the DFT conformational search.

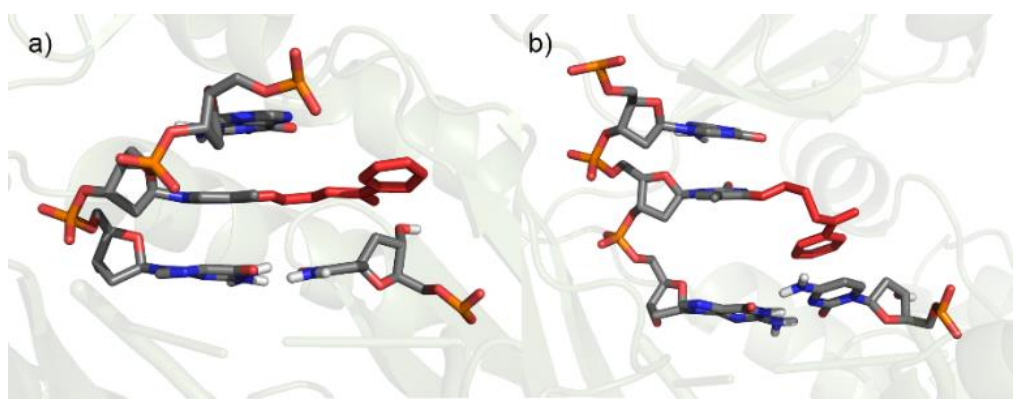

Figure S2: MD representative structure of the pol $\eta$ pre-insertion complex with a) O4-POB-T extended into the open pocket ( $>90 \%$ occupancy) or b) stacked with the 3 '-base pair (<10\% occupancy; nucleobase in grey, POB moiety in red). 

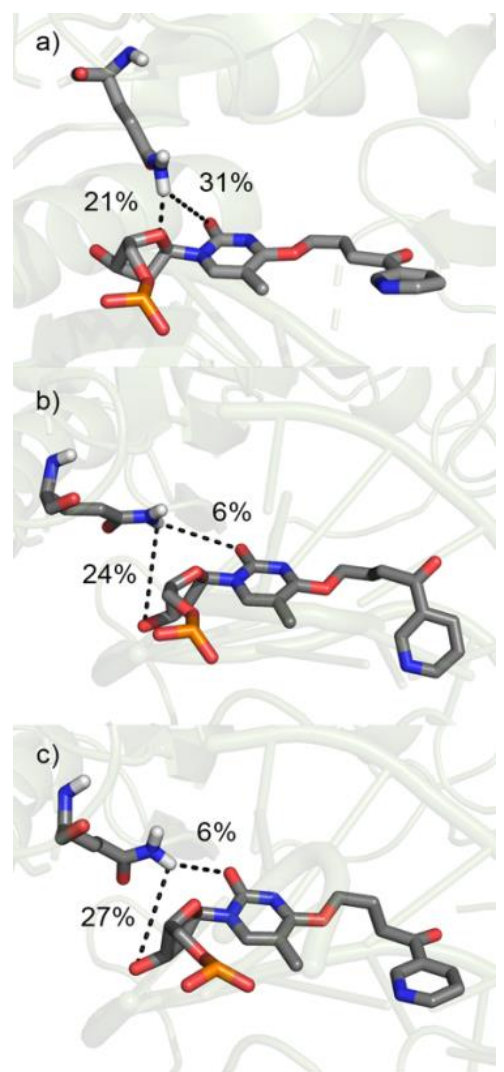

Figure S3: Hydrogen-bonding occupancies between O4-POB-T and Q38 in the pol $\eta$ a) pre-insertion complex, and ternary complex corresponding to b) dATP or c) dGTP insertion.

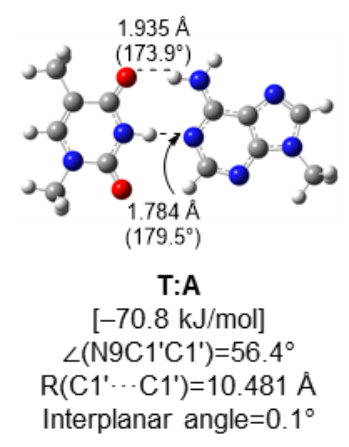

Figure S4: Key DFT structural parameters for the canonical T:A base pair $\left(\AA\right.$ and $\left.{ }^{\circ}\right)$ and the associated binding energy $[\mathrm{kJ} / \mathrm{mol}]$. 

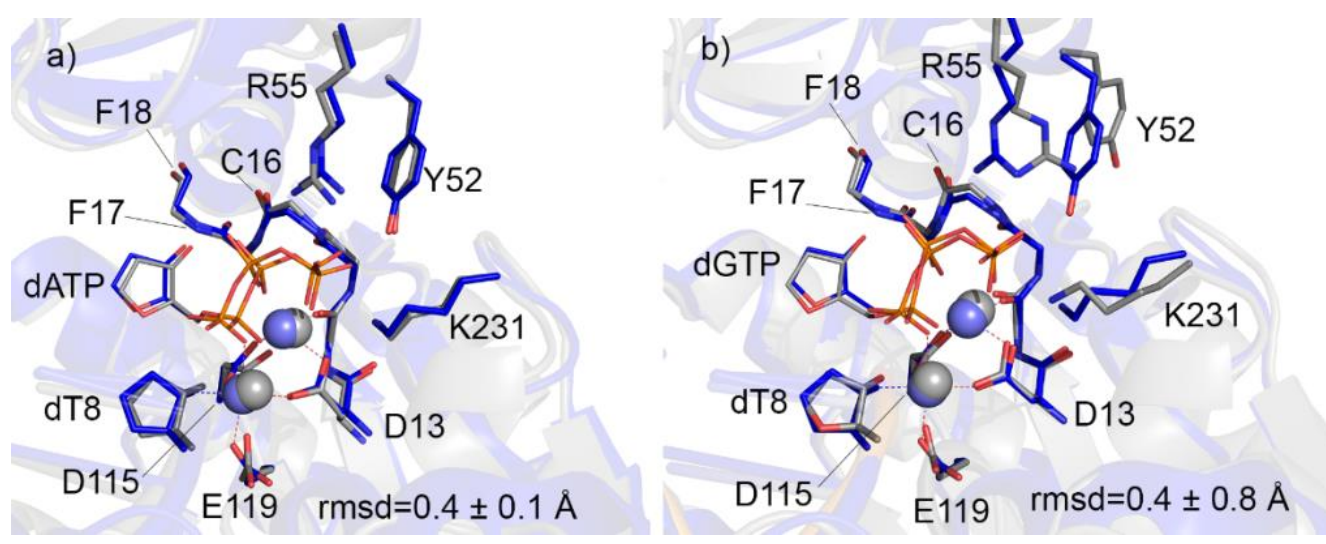

Figure S5: Overlay of X-ray crystal structure (PDB ID: 4ECS, blue) and MD representative structure (grey) for pol $\eta$ insertion of a) dATP and b) dGTP opposite O4-POB-T, highlighting alignment of key active site residues that interact with the incoming $\mathrm{dNTP}$.
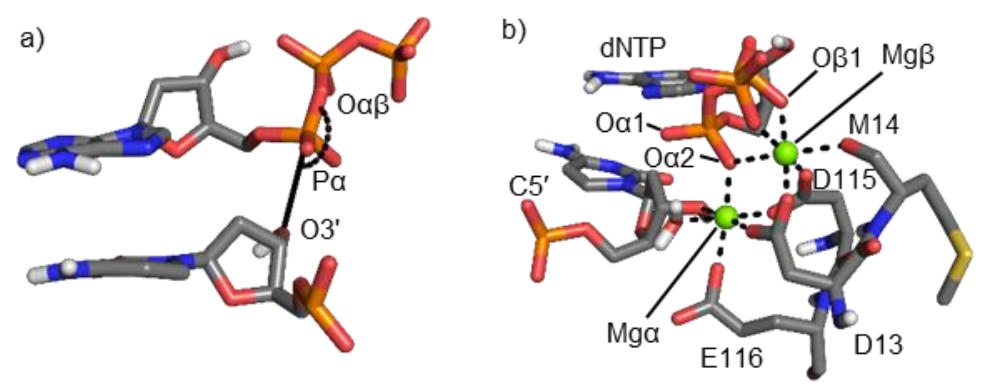

Figure S6: a) Definition of reaction parameters with respect to the incoming dNTP (top) and the corresponding $5^{\prime}$ base (bottom) and b) $\mathrm{Mg}^{2+}$ coordination required to promote catalytic activity of pol $\eta$.

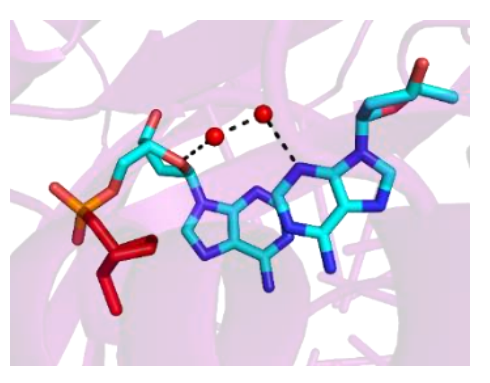

Figure S7: X-ray crystal structure (PDB ID: 4RNM) corresponding to pol $\eta$ insertion of dATP opposite an abasic site (red), highlighting the water chain proposed to explain "the A rule". 

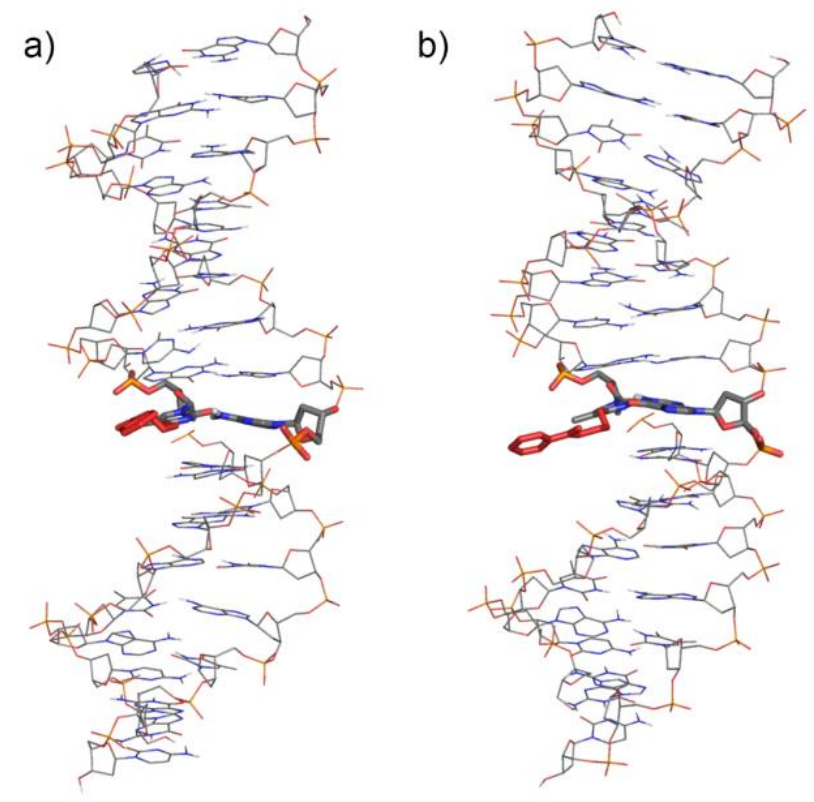

Figure S8: MD representative structure of DNA duplexes containing the a) O4-POB-T:A pair or b) O4POB-T:G mispair.

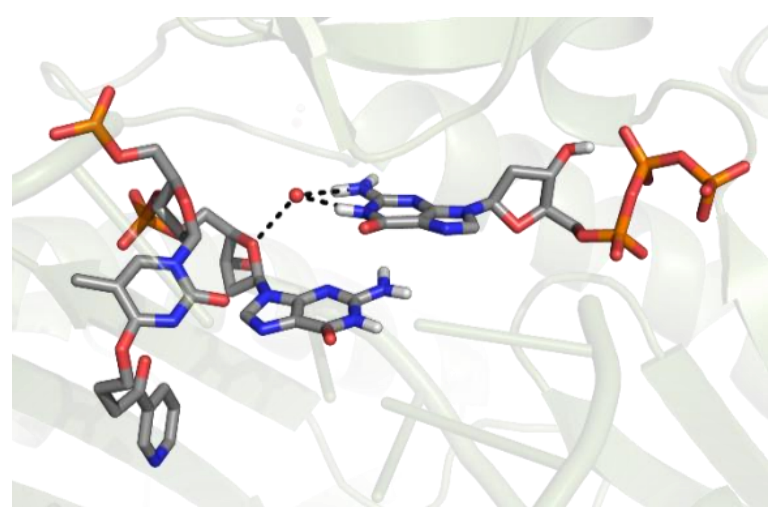

Figure S9: MD representative structure corresponding to pol $\eta$ insertion of dGTP partially stabilized by a water chain when O4-POB-T is located in the open pocket. 

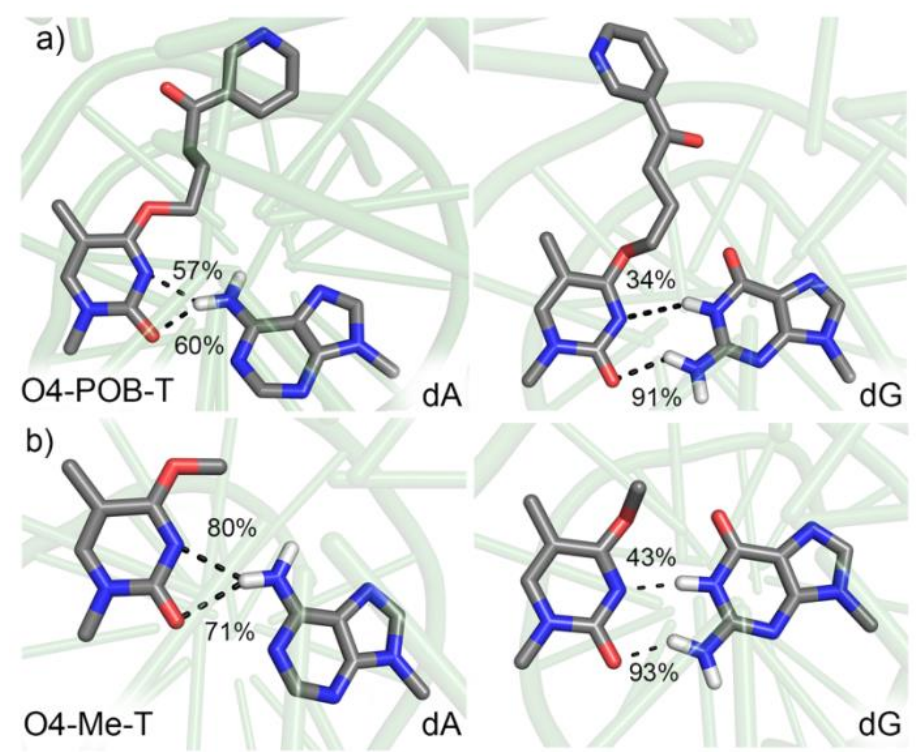

Figure S10: MD representative structure, including hydrogen-bond occupancies (\%), of the postextension DNA duplex corresponding to dATP (left) or dGTP (right) insertion opposite a) O4-POB-T or b) O4-Me-T.

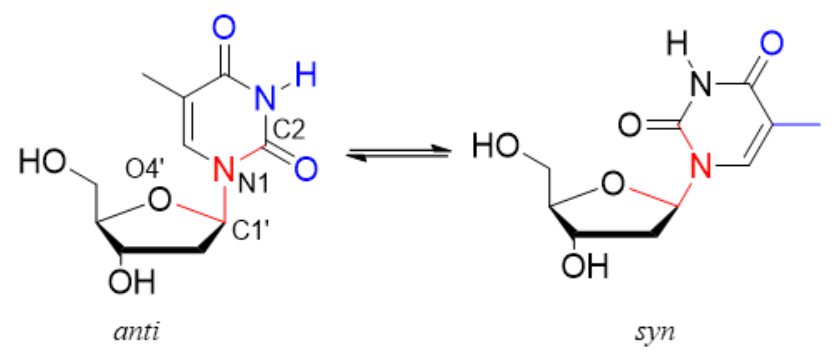

Figure S11: Rotation about the glycosidic bond $\left(\angle\left(\mathrm{O} 4^{\prime} \mathrm{C} 1^{\prime} \mathrm{N} 1 \mathrm{C} 2\right)\right.$, red $)$ in the canonical $\mathrm{T}$ nucleoside between the anti and syn conformations, as well as identification of the Watson-Crick (left) and Hoogsteen (right) hydrogen-bonding face (blue). 
Table S1: Atom types, atom names and partial charges for the O4-POB-T nucleobase used in the Hyperchem conformational search. ${ }^{a}$

\begin{tabular}{|c|c|c|}
\hline Atom Name & Atom Type & Charge \\
\hline N1 & $\mathrm{N}^{*}$ & -0.1311 \\
\hline C6 & $\mathrm{CM}$ & -0.0997 \\
\hline H6 & $\mathrm{H} 4$ & 0.201 \\
\hline $\mathrm{C} 5$ & $\mathrm{CM}$ & -0.1846 \\
\hline $\mathrm{C} 7$ & $\mathrm{CT}$ & -0.2316 \\
\hline H71 & $\mathrm{HC}$ & 0.0838 \\
\hline $\mathrm{H} 72$ & $\mathrm{HC}$ & 0.0838 \\
\hline $\mathrm{H} 73$ & $\mathrm{HC}$ & 0.0838 \\
\hline $\mathrm{C} 4$ & $\mathrm{CA}$ & 0.7792 \\
\hline $\mathrm{O} 4$ & OS & -0.358 \\
\hline N3 & $\mathrm{NC}$ & -0.7797 \\
\hline $\mathrm{C} 2$ & $\mathrm{C}$ & 0.7887 \\
\hline $\mathrm{O} 2$ & $\mathrm{O}$ & -0.5808 \\
\hline $\mathrm{C} 8$ & $\mathrm{CT}$ & -0.0221 \\
\hline H81 & $\mathrm{H} 1$ & 0.0966 \\
\hline H82 & H1 & 0.0966 \\
\hline C9 & $\mathrm{CT}$ & -0.0053 \\
\hline H91 & $\mathrm{HC}$ & 0.0353 \\
\hline H92 & $\mathrm{HC}$ & 0.0353 \\
\hline $\mathrm{C} 10$ & $\mathrm{CT}$ & 0.0044 \\
\hline H101 & $\mathrm{HC}$ & 0.0252 \\
\hline H102 & $\mathrm{HC}$ & 0.0252 \\
\hline $\mathrm{C} 11$ & $\mathrm{C}$ & 0.3549 \\
\hline O11 & $\mathrm{O}$ & -0.486 \\
\hline $\mathrm{C} 12$ & CA & -0.0915 \\
\hline $\mathrm{C} 13$ & $\mathrm{CA}$ & -0.0546 \\
\hline H13 & HA & 0.18 \\
\hline C14 & $\mathrm{CA}$ & -0.3497 \\
\hline H14 & HA & 0.1587 \\
\hline $\mathrm{C} 15$ & $\mathrm{CA}$ & 0.3553 \\
\hline H15 & $\mathrm{H} 4$ & 0.0699 \\
\hline N16 & $\mathrm{NC}$ & -0.5937 \\
\hline $\mathrm{C} 17$ & CA & 0.3099 \\
\hline H17 & $\mathrm{H} 4$ & 0.0899 \\
\hline $\mathrm{C} 1^{\prime}$ & $\mathrm{CT}$ & 0.1272 \\
\hline H1'1 & H1 & -0.0054 \\
\hline H1'2 & $\mathrm{H} 1$ & -0.0054 \\
\hline H1'3 & $\mathrm{H} 1$ & -0.0054 \\
\hline
\end{tabular}

${ }^{a}$ See Figure 1 (main text) for atom numbering. 
Table S2: Atom names and types, as well as associated partial charges for the O4-POB-T nucleotide used in MD simulations. ${ }^{a}$

\begin{tabular}{|c|c|c|}
\hline Atom Name & Atom Type & Charge \\
\hline $\mathrm{P}$ & $P$ & 1.1624 \\
\hline O5' & OS & -0.4935 \\
\hline O1P & $\mathrm{O} 2$ & -0.7628 \\
\hline $\mathrm{O} 2 \mathrm{P}$ & $\mathrm{O} 2$ & -0.77 \\
\hline O3' & $\mathrm{O}$ & -0.5447 \\
\hline $\mathrm{C}^{\prime}$ & CT & -0.0071 \\
\hline H5' & H1 & 0.0822 \\
\hline H5" & $\mathrm{H} 1$ & 0.0822 \\
\hline C4' & CT & 0.1368 \\
\hline H4' & H1 & 0.1077 \\
\hline O4' & OS & -0.4001 \\
\hline $\mathrm{C} 11^{\prime}$ & CT & 0.1772 \\
\hline H1' & $\mathrm{H} 2$ & 0.0842 \\
\hline C3' & CT & 0.1521 \\
\hline H3' & H1 & 0.0753 \\
\hline $\mathrm{C} 2^{\prime}$ & CT & -0.0596 \\
\hline $\mathrm{H} 2^{\prime}$ & $\mathrm{HC}$ & 0.0433 \\
\hline H2" & $\mathrm{HC}$ & 0.0433 \\
\hline N1 & $\mathrm{N}^{*}$ & -0.1353 \\
\hline C6 & $\mathrm{CM}$ & -0.1013 \\
\hline H6 & H4 & 0.2053 \\
\hline C5 & $\mathrm{CM}$ & -0.1881 \\
\hline C7 & CT & -0.2016 \\
\hline $\mathrm{H} 71$ & $\mathrm{HC}$ & 0.0759 \\
\hline H72 & $\mathrm{HC}$ & 0.0759 \\
\hline $\mathrm{H} 73$ & $\mathrm{HC}$ & 0.0759 \\
\hline $\mathrm{C} 4$ & CA & 0.78 \\
\hline $\mathrm{O} 4$ & OS & -0.3578 \\
\hline N3 & $\mathrm{NC}$ & -0.7838 \\
\hline $\mathrm{C} 2$ & $\mathrm{C}$ & 0.7944 \\
\hline $\mathrm{O} 2$ & $\mathrm{O}$ & -0.5824 \\
\hline C8 & $\mathrm{CT}$ & -0.0282 \\
\hline H81 & H1 & 0.0999 \\
\hline H82 & $\mathrm{H} 1$ & 0.0999 \\
\hline C9 & CT & -0.0094 \\
\hline H91 & $\mathrm{HC}$ & 0.0365 \\
\hline H92 & $\mathrm{HC}$ & 0.0365 \\
\hline $\mathrm{C} 10$ & CT & 0.0048 \\
\hline H10 & $\mathrm{HC}$ & 0.0252 \\
\hline H10 & $\mathrm{HC}$ & 0.0252 \\
\hline C11 & C & 0.3552 \\
\hline O11 & $\mathrm{O}$ & -0.486 \\
\hline $\mathrm{C} 12$ & CA & -0.0913 \\
\hline $\mathrm{C} 13$ & $\mathrm{CA}$ & -0.0542 \\
\hline H13 & HA & 0.1799 \\
\hline C14 & CA & 0.3096 \\
\hline H14 & $\mathrm{H} 4$ & 0.09 \\
\hline C15 & CA & -0.35 \\
\hline H15 & HA & 0.1589 \\
\hline C17 & CA & 0.355 \\
\hline H17 & $\mathrm{H} 4$ & 0.0701 \\
\hline N16 & $\mathrm{NC}$ & -0.5935 \\
\hline
\end{tabular}

${ }^{a}$ See Figure 1 (main text) for atom numbering. 
Table S3: Average backbone RMSD and associated standard deviation over MD simulations on pol $\eta$ ternary complexes and post-extension duplexes corresponding to dNTP insertion opposite O4-POB-T. ${ }^{a}$

\begin{tabular}{|c|c|c|c|c|c|c|c|c|}
\hline \multirow[b]{2}{*}{ Complex } & \multicolumn{2}{|c|}{ Replica 1} & \multicolumn{2}{|c|}{ Replica 2} & \multicolumn{2}{|c|}{ Replica 3} & \multicolumn{2}{|c|}{ Average $^{b}$} \\
\hline & $\begin{array}{c}\text { Avg } \\
\text { RMSD }\end{array}$ & SD & $\begin{array}{c}\text { Avg. } \\
\text { RMSD }\end{array}$ & SD & $\begin{array}{c}\text { Avg } \\
\text { RMSD }\end{array}$ & SD & $\begin{array}{c}\text { Avg } \\
\text { RMSD }\end{array}$ & SD \\
\hline dATP ternary $^{c}$ & 2.69 & 0.35 & 2.25 & 0.26 & 2.09 & 0.25 & 2.34 & 0.29 \\
\hline dATP ternary (DNA only) ${ }^{d}$ & 2.48 & 0.43 & 2.86 & 0.22 & 2.76 & 0.21 & 2.70 & 0.29 \\
\hline dATP post-extension duplex & 2.69 & 0.60 & 4.04 & 1.34 & 3.12 & 1.06 & 3.28 & 1.00 \\
\hline dGTP ternary $^{c}$ & 2.16 & 0.27 & 3.41 & 0.58 & 2.36 & 0.29 & 2.64 & 0.38 \\
\hline dGTP ternary (DNA only) ${ }^{d}$ & 1.98 & 0.61 & 2.34 & 0.50 & 2.17 & 0.52 & 2.16 & 0.54 \\
\hline dGTP post-extension duplex & 3.59 & 0.72 & 4.18 & 1.90 & 3.71 & 0.96 & 3.83 & 1.19 \\
\hline
\end{tabular}

${ }^{a}$ RMSDs are calculated with respect to the initial frame of the corresponding production simulation. ${ }^{b}$ Average and standard deviation over the three replicas. ${ }^{c}$ RMSD calculated for the entire polymerase-DNA complex. ${ }^{d}$ RMSD calculated for only DNA.

Table S4: Average distance and occupancy of $\mathrm{Mg}^{2+}$ coordinating residues throughout MD simulations on the pol $\eta$ ternary complex corresponding to dATP insertion opposite O4-POB-T. ${ }^{a}$

\begin{tabular}{|c|c|c|c|c|c|c|c|c|c|c|c|c|}
\hline \multirow[b]{2}{*}{ Interaction } & \multicolumn{3}{|c|}{ Replica 1} & \multicolumn{3}{|c|}{ Replica 2} & \multicolumn{3}{|c|}{ Replica 3} & \multicolumn{3}{|c|}{ Average $^{c}$} \\
\hline & $\begin{array}{c}\text { Dist. } \\
\text { (A) }\end{array}$ & $\begin{array}{l}\text { SD } \\
(\AA)\end{array}$ & $\begin{array}{c}\text { Occ. }^{b} \\
(\%)\end{array}$ & $\begin{array}{c}\text { Dist. } \\
(\AA)\end{array}$ & $\begin{array}{l}\text { SD } \\
(\AA)\end{array}$ & $\begin{array}{c}\text { Occ. }^{b} \\
(\%)\end{array}$ & $\begin{array}{c}\text { Dist. } \\
(\AA)\end{array}$ & $\begin{array}{l}\text { SD } \\
(\AA)\end{array}$ & $\begin{array}{c}\text { Occ. }^{b} \\
(\%)\end{array}$ & $\begin{array}{c}\text { Dist. } \\
\text { (§) }\end{array}$ & $\begin{array}{l}\text { SD } \\
(\AA)\end{array}$ & $\begin{array}{c}\text { Occ. }^{b} \\
(\%)\end{array}$ \\
\hline $\operatorname{Mg}(\alpha) \cdots \operatorname{dATP}(\mathrm{O} \alpha 2)$ & 2.1 & 0.1 & 99.7 & 2.1 & 0.1 & 99.7 & 2.1 & 0.1 & 99.8 & 2.1 & 0.1 & 99.7 \\
\hline $\operatorname{Mg}(\alpha) \cdots \mathrm{D} 13(\mathrm{O} \delta 1)$ & 2.0 & 0.0 & 100 & 2.0 & 0.0 & 100 & 2.0 & 0.0 & 100 & 2.0 & 0.0 & 100 \\
\hline $\operatorname{Mg}(\alpha) \cdots E 116(O € 2)$ & 1.9 & 0.0 & 100 & 1.9 & 0.0 & 100 & 1.9 & 0.0 & 100 & 1.9 & 0.0 & 100 \\
\hline $\operatorname{Mg}(\alpha) \cdots \mathrm{D} 115(\mathrm{O} \delta 1)$ & 2.0 & 0.1 & 100 & 2.0 & 0.1 & 100 & 2.0 & 0.1 & 100 & 2.0 & 0.1 & 100 \\
\hline $\operatorname{Mg}(\alpha) \cdots 5^{\prime}-\mathrm{C}\left(\mathrm{O}^{\prime}\right)$ & 2.2 & 0.1 & 99.6 & 2.2 & 0.1 & 99.4 & 2.2 & 0.1 & 99.7 & 2.2 & 0.1 & 99.6 \\
\hline $\operatorname{Mg}(\alpha) \cdots$ Water $(O)$ & 2.1 & 0.1 & 100 & 2.1 & 0.1 & 100 & 2.1 & 0.1 & 100 & 2.1 & 0.1 & 100 \\
\hline $\operatorname{Mg}(\beta) \cdots \mathrm{D} 13(\mathrm{O} \delta 2)$ & 2.1 & 0.1 & 100 & 2.1 & 0.1 & 100 & 2.1 & 0.1 & 100 & 2.1 & 0.1 & 100 \\
\hline $\operatorname{Mg}(\beta) \cdots \mathrm{M} 17(\mathrm{O})$ & 2.0 & 0.0 & 100 & 2.0 & 0.0 & 100 & 2.0 & 0.0 & 100 & 2.0 & 0.0 & 100 \\
\hline $\operatorname{Mg}(\beta) \cdots D 115(\mathrm{O} \delta 2)$ & 2.0 & 0.0 & 100 & 2.0 & 0.0 & 100 & 2.0 & 0.0 & 100 & 2.0 & 0.0 & 100 \\
\hline $\operatorname{Mg}(\beta) \cdots \operatorname{dATP}(\mathrm{O} \gamma 1)$ & 1.9 & 0.0 & 100 & 1.9 & 0.0 & 100 & 1.9 & 0.0 & 100 & 1.9 & 0.0 & 100 \\
\hline $\operatorname{Mg}(\beta) \cdots \mathrm{dATP}(\mathrm{O} \beta 2)$ & 1.9 & 0.0 & 100 & 1.9 & 0.0 & 100 & 1.9 & 0.0 & 100 & 1.9 & 0.0 & 100 \\
\hline $\operatorname{Mg}(\beta) \cdots \operatorname{dATP}(\mathrm{O} \alpha 2)$ & 2.2 & 0.2 & 90.3 & 2.3 & 0.2 & 80.1 & 2.3 & 0.2 & 84.2 & 2.3 & 0.2 & 84.9 \\
\hline
\end{tabular}

${ }^{a}$ See Figure S6a for atomic numbering. ${ }^{b}$ Occupancies were determined according to a distance cutoff of $2.5 \AA$. ${ }^{c}$ Average and standard deviation over the three replicas. 
Table S5: Average base-pair hydrogen-bonding distances $(\AA)$ and angles (deg.) between O4-POB-T and an opposing A or G over MD simulations on ternary complexes and post-extension duplexes.

\begin{tabular}{|c|c|c|c|c|c|c|c|c|c|}
\hline & \multirow[b]{2}{*}{ Hydrogen Bond } & \multicolumn{2}{|c|}{ Replica 1} & \multicolumn{2}{|c|}{ Replica 2} & \multicolumn{2}{|c|}{ Replica 3} & \multicolumn{2}{|c|}{ Average $^{a}$} \\
\hline & & $\begin{array}{l}\text { Dist. } \\
(\AA)\end{array}$ & $\begin{array}{l}\text { Angle } \\
\text { (deg.) }\end{array}$ & $\begin{array}{c}\text { Dist. } \\
\text { (̊) }\end{array}$ & $\begin{array}{l}\text { Angle } \\
\text { (deg.) }\end{array}$ & $\begin{array}{c}\text { Dist. } \\
(\AA)\end{array}$ & $\begin{array}{l}\text { Angle } \\
\text { (deg.) }\end{array}$ & $\begin{array}{l}\text { Dist. } \\
(\AA)^{a}\end{array}$ & $\begin{array}{l}\text { Angle } \\
\text { (deg.) }\end{array}$ \\
\hline \multirow{2}{*}{$\begin{array}{c}\text { dATP } \\
\text { post-extension } \\
\text { duplex }\end{array}$} & N3(O4-POB-T) $\cdots($ N6-H)A & 3.1 & 141.5 & 3.1 & 131.7 & 3.1 & 140.8 & 3.1 & 138.0 \\
\hline & $\mathrm{O} 2(\mathrm{O} 4-\mathrm{POB}-\mathrm{T}) \cdots(\mathrm{N} 6-\mathrm{H}) \mathrm{A}$ & 3.1 & 156.8 & 3.1 & 153.1 & 3.1 & 154.7 & 3.1 & 154.9 \\
\hline \multirow{3}{*}{$\begin{array}{c}\text { dGTP } \\
\text { ternary } \\
\text { complex }\end{array}$} & $\mathrm{O} 2(\mathrm{O} 4-\mathrm{POB}-\mathrm{T}) \cdots(\mathrm{N} 2-\mathrm{H}) \mathrm{G}$ & 2.9 & 149.1 & 3.0 & 150.2 & 3.0 & 154.7 & 3.0 & 151.3 \\
\hline & $\mathrm{O} 2(\mathrm{O} 4-\mathrm{POB}-\mathrm{T}) \cdots(\mathrm{N} 1-\mathrm{H}) \mathrm{G}$ & 3.3 & 155.8 & 3.0 & 144.4 & 3.0 & 145.6 & 3.0 & 145.6 \\
\hline & $\mathrm{N} 3(\mathrm{O} 4-\mathrm{POB}-\mathrm{T}) \cdots(\mathrm{N} 2-\mathrm{H}) \mathrm{G}$ & 3.2 & 154.7 & 3.2 & 156.9 & 3.2 & 155.5 & 3.2 & 156.3 \\
\hline \multirow{3}{*}{$\begin{array}{c}\text { dGTP } \\
\text { post-extension } \\
\text { duplex }\end{array}$} & $\mathrm{O} 2(\mathrm{O} 4-\mathrm{POB}-\mathrm{T}) \cdots(\mathrm{N} 2-\mathrm{H}) \mathrm{G}$ & 2.8 & 153.2 & 2.9 & 154.0 & 2.8 & 153.5 & 2.9 & 153.6 \\
\hline & $\mathrm{N} 3(\mathrm{O} 4-\mathrm{POB}-\mathrm{T}) \cdots(\mathrm{N} 1-\mathrm{H}) \mathrm{G}$ & 3.2 & 156.1 & 3.1 & 143.5 & 3.1 & 156.0 & 3.1 & 151.9 \\
\hline & $\mathrm{N} 3(\mathrm{O} 4-\mathrm{POB}-\mathrm{T}) \cdots(\mathrm{N} 2-\mathrm{H}) \mathrm{G}$ & 3.1 & 154.3 & 3.1 & 142.9 & 3.1 & 154.2 & 3.1 & 150.5 \\
\hline
\end{tabular}

${ }^{a}$ Average and standard deviation over the three replicas.

Table S6: Average distance and occupancy of $\mathrm{Mg}^{2+}$ coordinating residues throughout MD simulations on the pol $\eta$ ternary complex corresponding to dGTP insertion opposite O4-POB-T. ${ }^{a}$

\begin{tabular}{|c|c|c|c|c|c|c|c|c|c|c|c|c|}
\hline \multirow[b]{2}{*}{ Interaction } & \multicolumn{3}{|c|}{ Replica 1} & \multicolumn{3}{|c|}{ Replica 2} & \multicolumn{3}{|c|}{ Replica 3} & \multicolumn{3}{|c|}{ Average $^{c}$} \\
\hline & $\begin{array}{c}\text { Dist. } \\
\text { (Å) }\end{array}$ & $\begin{array}{l}\text { SD } \\
(\AA)\end{array}$ & $\begin{array}{c}\text { Occ. }^{b} \\
(\%)\end{array}$ & $\begin{array}{c}\text { Dist. } \\
\text { (Å) }\end{array}$ & $\begin{array}{l}\text { SD } \\
(\AA)\end{array}$ & $\begin{array}{c}\text { Occ. }^{b} \\
(\%)\end{array}$ & $\begin{array}{c}\text { Dist. } \\
\text { (Å) }\end{array}$ & $\begin{array}{l}\text { SD } \\
(\AA)\end{array}$ & $\begin{array}{c}\text { Occ. }^{b} \\
(\%)\end{array}$ & $\begin{array}{c}\text { Dist. } \\
\text { (Å) }\end{array}$ & $\begin{array}{l}\text { SD } \\
(\AA)\end{array}$ & $\begin{array}{c}\text { Occ. }^{b} \\
(\%)\end{array}$ \\
\hline $\operatorname{Mg}(\alpha) \cdots \mathrm{dGTP}(\mathrm{O} \alpha 2)$ & 2.4 & 0.2 & 75 & 2.4 & 0.2 & 69 & 2.4 & 0.2 & 75 & 2.4 & 0.2 & 73 \\
\hline $\operatorname{Mg}(\alpha) \cdots \mathrm{D} 13(\mathrm{O} \delta 1)$ & 2.0 & 0.0 & 100 & 2.0 & 0.0 & 100 & 2.0 & 0.2 & 100 & 2.0 & 0.1 & 100 \\
\hline $\operatorname{Mg}(\alpha) \cdots E 116(\mathrm{O} \in 2)$ & 2.4 & 0.9 & 74 & 1.9 & 0.0 & 100 & 1.9 & 0.2 & 100 & 2.1 & 0.4 & 91 \\
\hline $\operatorname{Mg}(\alpha) \cdots \mathrm{D} 115(\mathrm{O} \delta 1)$ & 2.0 & 0.0 & 100 & 2.0 & 0.0 & 100 & 2.0 & 0.2 & 100 & 2.0 & 0.1 & 100 \\
\hline $\operatorname{Mg}(\alpha) \cdots 5^{\prime}-\mathrm{C}\left(\mathrm{O}^{\prime}\right)$ & 2.1 & 0.1 & 100 & 2.1 & 0.1 & 100 & 2.2 & 0.2 & 94 & 2.2 & 0.1 & 98 \\
\hline $\operatorname{Mg}(\alpha) \cdots$ Water $(\mathrm{O})$ & 2.1 & 0.1 & 100 & 2.1 & 0.1 & 100 & 2.1 & 0.1 & 100 & 2.1 & 0.1 & 100 \\
\hline $\operatorname{Mg}(\beta) \cdots \mathrm{D} 13(\mathrm{O} \delta 2)$ & 2.1 & 0.1 & 100 & 2.1 & 0.1 & 100 & 2.1 & 0.3 & 100 & 2.1 & 0.1 & 100 \\
\hline $\operatorname{Mg}(\beta) \cdots \operatorname{M17}(\mathrm{O})$ & 2.0 & 0.0 & 100 & 1.9 & 0.0 & 100 & 1.9 & 0.2 & 100 & 1.9 & 0.1 & 100 \\
\hline $\operatorname{Mg}(\beta) \cdots \mathrm{D} 115(\mathrm{O} \delta 2)$ & 2.0 & 0.0 & 100 & 2.0 & 0.0 & 100 & 2.0 & 0.2 & 100 & 2.0 & 0.1 & 100 \\
\hline $\operatorname{Mg}(\beta) \cdots \mathrm{dGTP}(\mathrm{O} \gamma 1)$ & 1.9 & 0.0 & 100 & 1.9 & 0.0 & 100 & 1.9 & 0.1 & 100 & 1.9 & 0.0 & 100 \\
\hline $\operatorname{Mg}(\beta) \cdots \mathrm{dGTP}(O \beta 2)$ & 2.4 & 0.1 & 88 & 2.5 & 0.2 & 60 & 2.6 & 0.7 & 61 & 2.5 & 0.3 & 70 \\
\hline $\operatorname{Mg}(\beta) \cdots \mathrm{dGTP}(\mathrm{O} \alpha 2)$ & 2.1 & 0.1 & 100 & 2.1 & 0.1 & 100 & 2.2 & 0.5 & 93 & 2.2 & 0.2 & 98 \\
\hline
\end{tabular}

${ }^{a}$ See Figure S6a for atomic numbering. ${ }^{b}$ Occupancies were determined according to a distance cutoff of $2.5 \AA .{ }^{c}$ Average and standard deviation over the three replicas. 